\title{
Brillouin distributed sensing assisted by Brillouin amplification of pump pulses
}

\author{
Javier Urricelqui, Mikel Sagues, Alayn Loayssa \\ Departamento de Ingeniería Eléctrica y Electrónica \\ Universidad Pública de Navarra \\ Pamplona 31006, Spain \\ alayn.loayssa@unavarra.es
}

\begin{abstract}
We demonstrate, for the first time to our knowledge, the extension of the measurement range of Brillouin distributed sensors (BDS) by using a distributed Brillouin amplifier (DBA) to compensate the attenuation of the sensor's pump pulses. The technique is based on injecting an extra DBA pump wave in the fiber to generate an additional Brillouin interaction to that used for sensing. Moreover, the bandwidth of the DBA is tailored to fit that of the pump pulses by deploying a wavelength modulation of the DBA pump that is synchronized to the pump. Experimental proof-of-concept results demonstrate a fivefold enhancement of the measurement range of a BDS, from $10.36 \mathrm{~km}$ to more than $50 \mathrm{~km}$ in this particular case. Moreover, it is found that the use of the DBA does not introduce any significant penalty in the detection signal-to-noise ratio, highlighting the potential of the technique to provide much larger sensing lengths.
\end{abstract}

Keywords-Brillouin distributed sensors, Brillouin optical time domain analysis, Brillouin amplification.

\section{INTRODUCTION}

Brillouin distributed fiber optic sensors, particularly of the Brillouin optical time-domain analysis (BOTDA) type, are a powerful tool for the monitoring of large structures. For instance, they find application in civil and geotechnical engineering structures, in the oil and gas industry, for the monitoring of large pipelines, or in the electrical grid, for the assessment of aerial and underground high voltage cables. All these fields require long measurements lengths.

However, fiber attenuation establishes a fundamental limit to the length of fiber that can be sensed in a BOTDA sensor because the amplitude of the pump pulses is reduced as they propagate along the fiber, which leads to small Brillouin gain at distant locations. As a consequence, the signal to noise ratio (SNR) of the detected probe signal becomes too small to provide measurements with the required precision. The SNR reduction can be compensated to some degree by the use of signal averaging, but at the cost of increasing the measurement time, which becomes impractically large. Moreover, this deterioration of performance cannot be counteracted by indefinitely increasing the pulse power because this is ultimately limited by the onset of nonlinear effects such as modulation instability and Raman scattering [1].

The obvious solution is to amplify the pulses to compensate the attenuation of the fiber. This has been demonstrated by the use of erbium-doped fiber amplifiers (EDFA) as pulse repeaters along the fiber length [2]. However, distant EDFAs need to be powered, whereas an all-passive sensing network is much more attractive and practical. A more elegant solution is to use distributed Raman amplification (DRA) by injecting a Raman pump in the sensing fiber [3] [4]. However this requires the use of very high power (of the order of Watts) in the fiber, which can become an eye-safety concern in the installation and operation of real systems. Moreover, with DRA the relative intensity noise of the Raman pump laser is translated to the detected signal, significantly degrading measurement performance [4].

In this work we demonstrate, for the first time to our knowledge, an alternative and more efficient approach: the use of distributed Brillouin amplification (DBA) to amplify the pump pulses as they travel along the fiber. This has the advantage of requiring much lower pump power than with DRA, because stimulated Brillouin scattering is intrinsically much more efficient than stimulated Raman scattering, with just milliwatts of pump power required to obtain large gain in typical lengths of fiber. Moreover, we introduce a method to precisely tailor the generated gain bandwidth spectrum to the pulse that is being amplified. This contrasts to the use of DRA, which provides a large gain bandwidth (typically tens of nanometers) that is largely wasted in this application to provide gain to BOTDA pump pulses that are narrowband.

\section{FUNDAMENTALS OF THE TECHNIQUE}

Fig. 1 schematically depicts the fundamentals of the technique. This is illustrated with a conventional BOTDA setup that uses a single laser to generate the pump pulses and the $\mathrm{CW}$ probe wave. However, the method is perfectly applicable to BOTDA schemes using other configurations, such as those based on using two separated lasers to generate pump and probe [2]. In the particular setup of Fig. 1, a pulse generator and optical switch are used in the upper branch to generate the pump pulses. In the lower branch, a tunable-wavelength probe is generated using the modulation sidebands obtained by driving a Mach-Zehnder electro-optic modulator with the signal from a microwave generator that outputs frequencies close to the Brillouin frequency shift (BFS) of the sensing fiber.

The only addition to this well-known scheme that is required by our technique is the incorporation of an additional laser (DBA amplification pump) whose output is also coupled into the sensing fiber and counter-propagated to the pump pulses. 


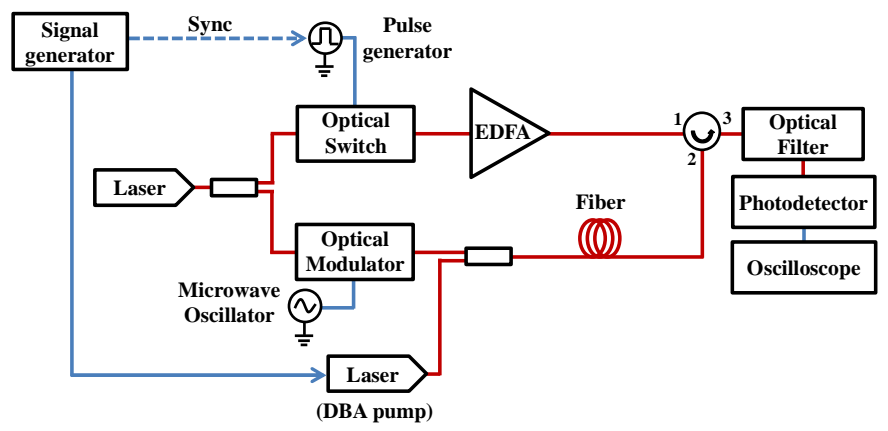

Fig. 1. Fundamentals of the technique.

Fig. 2(a) highlights the spectra of the optical waves that are simultaneously present in the sensing fiber. Notice that we have two simultaneous Brillouin interactions: the conventional BOTDA interaction between the pump pulses and the CW probe wave, and an additional interaction between the DBA pump and the pump pulses. The latter is responsible for the amplification of the pump pulses as they travel along the fiber. This requires that the DBA pump wave is offset from the pump pulse by an optical frequency difference that is around the mean Brillouin frequency shift (BFS) of the sensing fiber.

However, the Brillouin gain spectrum generated by a $\mathrm{CW}$ DBA pump would be very narrow, just around the $30 \mathrm{MHz}$ $(\sim 0.3 \mathrm{pm})$ intrinsic Brillouin linewidth, which, for instance, would not be enough to amplify the pump pulses required for 1-m spatial resolution. This pulses would have around 10-ns duration and a spectral bandwidth of a few hundreds megahertz depending on the pulse shape.

Therefore, there is a need to increase the DBA bandwidth beyond the natural Brillouin linewidth to properly amplify all optical spectral components of the pump pulse. In our technique we achieve this by modulating the wavelength of the DBA pump wave, as it is schematically depicted in Fig. 2(a). This modulation is performed by directly driving the current applied to the semiconductor laser used to generate the DBA pump, so as to take advantage of the laser's chirp, which translates this direct current modulation into optical frequency modulation. The current modulation is provided by an electrical waveform generator, which is synchronized to the pulse generator driving the optical switch to generate the pump pulses of the BOTDA.

The net result of this modulation of the DBA pump is that along the fiber the pump pulses experience a position-dependent Brillouin gain generated by the DBA pump. Fig. 2(a) schematically depicts this situation. The wavelength modulation of the DBA pump makes the wavelength location of the Brillouin gain spectrum to vary over time. Moreover, this time change translates into a change of the Brillouin spectrum detuning along the fiber as the DBA pump propagates. Indeed, the detuning of the pump pulse wavelength from the center of the Brillouin gain spectrum, $\Delta v$, at each position along the fiber is given by:

$$
\Delta \mathrm{v}(\mathrm{z})=\mathrm{v}_{\mathrm{P}}-\mathrm{v}_{\mathrm{L}}(\mathrm{z})-\mathrm{BFS}(\mathrm{z})
$$

where $v_{P}$ is the wavelength of the pump pulses and $v_{L}$ is the local wavelength of the DBA pump. Note that the latter changes due to the wavelength modulation that is impressed upon its laser source. Moreover, each pulse always experiences the same Brillouin spectrum detuning at each particular location due to the fact that the DBA pump wavelength modulation and the pump pulses generation are synchronized. Fig. 2(b) depicts the calculated local Brillouin spectrum that is experienced by the pump pulses at each location of a $50-\mathrm{km}$ fiber for a triangular modulation of the DBA pump with a frequency of $80 \mathrm{KHz}$ and a peak frequency deviation of $125 \mathrm{MHz}$. A zoom of the $\mathrm{z}$ axis showing just the first $5 \mathrm{~km}$ of the fiber is used in Fig. 2(b) to highlight that the profile of the detuning of the Brillouin spectrum along the fiber indeed follows the same triangular profile of the modulation.

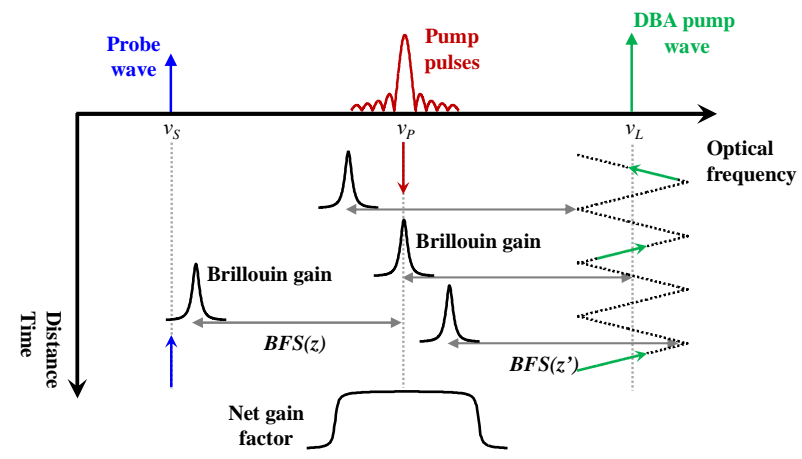

(a)

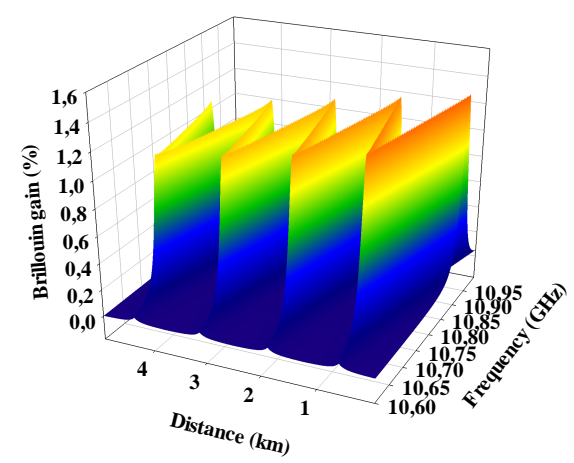

(b)

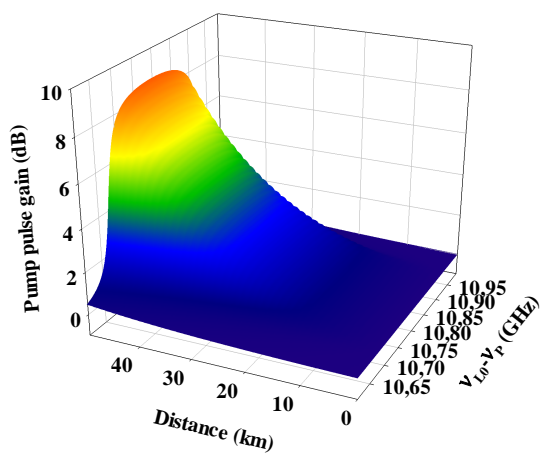

(c)

Fig. 2. (a) Spectra of optical waves in the fiber. (b) Local Brillouin gain experienced by the pump pulses at each location and (c) total gain experienced by the pulses in their propagation for each location. $v_{\mathrm{L} 0}$ is the wavelength of the DBA pump when no modulation is applied. Simulation's parameters are: Brillouin gain $1.110^{-11} \mathrm{~m} / \mathrm{W}$, Brillouin linewidth $30 \mathrm{MHz}$, effective area is $810^{11} \mathrm{~m}^{2}$ and the injected optical pump and probe power are $100 \mathrm{~mW}$ and $4.7 \mathrm{~mW}$ 
Note that a uniform BFS fiber is assumed in these calculations. Fig. 2(c) depicts the total gain accumulated by the pump pulses from their entry in the fiber (at $\mathrm{z}=0$ ) as they propagate to each particular position. This total gain is the result of the integration of the position dependent gain provided by the Brillouin spectrum at each $z$. These simulations have been performed by solving the coupled differential equations that govern Brillouin interaction in the fiber assuming steady-state conditions (pulse duration>10ns) and typical parameters for Brillouin interaction in the fiber (see caption of Fig. 2) [5].

Finally, notice that the choice of wavelength modulation shape and frequency impacts the bandwidth and the spectral flatness of the resulting gain spectrum of the DBA for each location. A triangular shape has been applied to provide a convenient response that amplifies all spectral components of the BOTDA pump pulses without adding distortion.

\section{EXPERIMENTAL DEMONSTRATION}

The basic setup described in Fig. 1 was assembled using a 1550-nm 10-mW laser source, a semiconductor optical amplifier as switch to generate 15-ns pump pulses, a Mach-Zehnder electro-optic modulator and a $125-\mathrm{MHz}$ photodetector. The Mach-Zehnder modulator was driven by a microwave synthesizer and biased at minimum transmission in order to generate a double-sideband suppressed carrier signal. This provides a double probe wave, which has been shown to greatly compensate non-local effects [6]. Each of these probe waves is launched in the fiber with a power of $-8 \mathrm{dBm}$. The peak pulse power injected into the fiber was $22.4 \mathrm{dBm}$. Furthermore, a polarization switch was also added, as it is customary in BOTDA setups, to eliminate the polarization-dependent response of Brillouin interaction. Before detecting the optical signal, the double probe wave was filtered out to operate in gain mode and the resulting component was pre-amplified by an EDFA. The deployed fiber was a $50-\mathrm{km}$ spool of standard single mode fiber.

In addition to the basic components of the BOTDA enumerated above, extra components were added to implement our technique for range extension using DBA: a DFB laser to serve as DBA pump and a signal generator to create the wavelength modulation. Note that the optical filter used to retain just one of the received probe sidebands, also filters out the remaining of the DBA laser power. The pump power for DBA injected in the fiber was $4.7 \mathrm{~mW}$. Moreover, the current of the DBA pump laser wavelength was modulated with a triangular electrical signal with a frequency of $80 \mathrm{KHz}$ and an amplitude adjusted to obtain a peak optical frequency deviation of $125 \mathrm{MHz}$. The resultant wavelength modulation was not perfectly triangular, but was slightly deformed due to the characteristics of the laser chirp [7]. This could be improved employing well-known methods to linearize the chirp response [7], but this was not necessary for the experiments outlined here.

Fig. 3 compares the distributed Brillouin gain spectra measured along the fiber when the DBA laser is switched off (Fig. 3(a)), and hence the system is a conventional BOTDA, and when it is switched on to become a Brillouin-assisted BOTDA (Fig. 3(b)). With the DBA off the spectra measured along the fiber gradually reduce their amplitude due to the progressive attenuation of the pump pulse as it propagates along the fiber. In contrast, when the DBA is on, the spectra amplitude initially decays but then starts to recover by mid-span. This is due to the effect of the gain generated by the DBA pump starting to kick-in. The latter is injected into the end of the fiber opposite to the entrance of the pump pulses and the gain that it generates is proportional to its power. Therefore, the pump pulses find amplification where they need it most: at the final segment of the fiber where they have experienced more attenuation.

The effect of the DBA is even more apparent in Fig. 4, where two BOTDA traces are compared. A logarithmic scale is deployed, hence the conventional BOTDA trace is seen to linearly fall with a slope given by the fiber's attenuation coefficient $(0.184 \mathrm{~dB} / \mathrm{km})$. Therefore, at $50 \mathrm{~km}$ the detected signal falls by $9.2 \mathrm{~dB}$. Considering that detected SNR is proportional to the square of the detected amplitude, the SNR degradation at the end of the fiber compared to the start is double the value in decibels of the extra attenuation of the pulse, i.e., $18.4 \mathrm{~dB}$. With the DBA-assisted BOTDA the worst location in terms of attenuation is around mid-span. Nevertheless, the maximum attenuation at that location is reduced to $2.95 \mathrm{~dB}$ and hence, the maximum SNR degradation to $5.9 \mathrm{~dB}$.

Nonetheless, the most significant improvement obtained with the presented technique is observed at the final section of the fiber, where the conventional BOTDA technique obtains

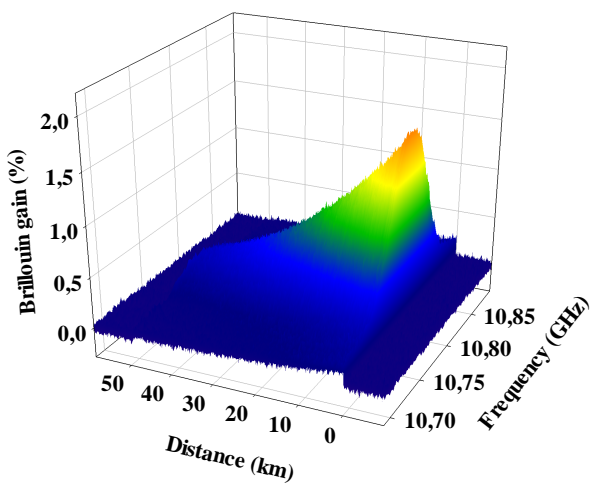

(a)

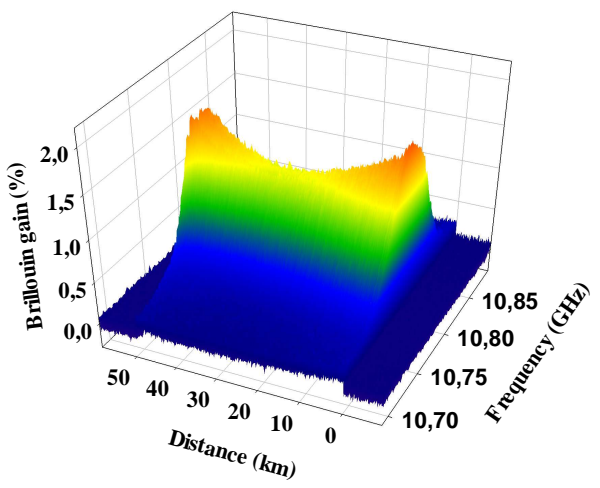

(b)

Fig. 3. Measured distribution of the Brillouin spectra when the DBA is turned off (a) and on (b). 
the worst results. Figure 5 compares the measured Brillouin spectra at the first and final sections of the fiber using the DBA-assisted BOTDA. Notice that the measured Brillouin gain at the final section is similar in amplitude to that measured at the first km. For this particular measurement, the pulse gain along the fiber was set to $8.9 \mathrm{~dB}$.

Furthermore, if we compare the measured spectra at the first and last sections with the DBA on, it is clear that there is not noticeable degradation of the SNR between both measurements, as both have almost the same amplitude and noise level. This is confirmed in Fig. 6, where the evolution of the SNR was measured for both the conventional and the DBA-assisted BOTDA. Moreover, note that the measured SNR at the start of the fiber is identical turning on and off the DBA (both measurements were performed with the same number of averages at the oscilloscope). As a consequence, it can be concluded that for these experiments the use of the DBA does not lead to any significant SNR penalty. At the start of the fiber, the SNR for both systems is identical. However, once the pump pulse is amplified by the DBA, the SNR grows instead of linearly decaying along the fiber as it happens in the conventional system.

Finally, we would like to remark that the use of DBA greatly extends the measurement range of the sensor. The worst SNR for the $50-\mathrm{km}$ DBA-assisted BOTDA is $32.13 \mathrm{~dB}$. To obtain this same SNR value with the conventional BOTDA the sensing distance needs to be limited to just $10.36 \mathrm{~km}$. This means that the DBA-assisted BOTDA brings a fivefold improvement in the measurement range for identical sensor performance.

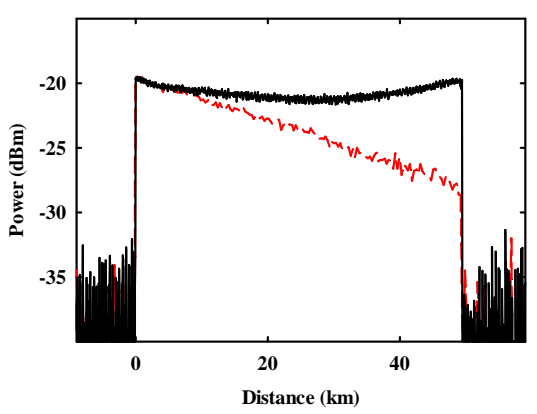

Fig. 4. Measured BOTDA traces when DBA is switched on (black-solid line) and off (red-dashed line).

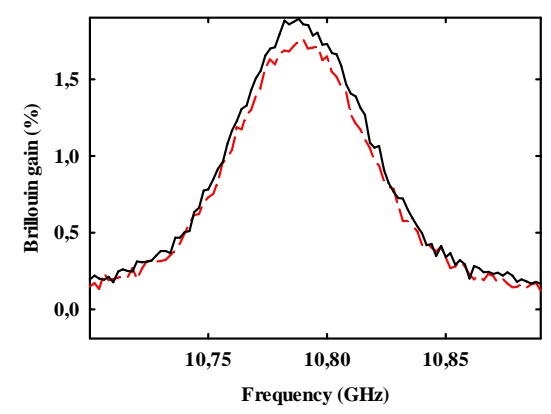

Fig. 5. Measured Brillouin spectra at the first (black-solid line) and final (red-dashed line) location of the fiber using the DBA-assisted technique.

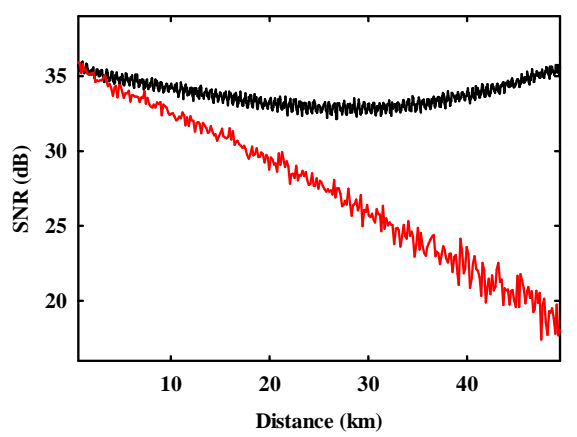

Fig. 6. SNR evolution for the DBA-assisted technique (black-solid line) and the conventional BOTDA sensor (red-dashed line).

\section{CONCLUSIONS}

In this work, we have presented a novel technique to increase the measurement range of conventional BOTDA sensors. It is based on the amplification of the pump pulses used to interrogate the sensing fiber by deploying a distributed Brillouin amplifier. The technique has been theoretically studied and proof-of-concept experiments have confirmed a fivefold extension of the measurement range compared to the conventional BOTDA. In addition, the study of the SNR points out that the deployment the DBA-assisted BOTDA technique does not introduce extra noise sources into the detected probe wave, solving one of the fundamental limits of the technique based on DRA.

\section{ACKNOWLEDGMENT}

The authors wish to acknowledge the financial support from the Universidad Pública de Navarra and Spanish Ministerio de Ciencia e Innovación through the projects TEC2010-20224C02-01 and TEC2013-47264-C2-2-R.

\section{REFERENCES}

[1] S. M. Foaleng, and L. Thévenaz, "Impact of Raman scattering and modulation instability on the performance of Brillouin sensors," Proc. of SPIE, vol. 7753, May 2011.

[2] Y. Dong, L. Chen, and X. Bao, "Extending the sensing range of Brillouin optical time-domain analysis combining frequency-division multiplexing and in-line EDFAs," J. Lightw. Technol., vol. 30, pp. 1161-1167, April 2012.

[3] A. Zornoza, et al., "Long-range hybrid network with point and distributed Brillouin sensors using Raman amplification," Opt. Express, vol. 18, pp. 9531-9541 (2010).

[4] X. Angulo-Vinuesa, et al., "Raman-assisted Brillouin distributed temperature sensor over $100 \mathrm{~km}$ featuring $2 \mathrm{~m}$ resolution and $1.2^{\circ} \mathrm{C}$ uncertainty," J. Lightw. Technol., vol. 30, pp. 1060-1065, Apr. 2012.

[5] X. Bao, J. Dhliwayo, N. Heron, D. J. Webb, and D. A. Jackson, "Experimental and theoretical studies on a distributed temperature sensor based on Brillouin scattering" J. Lightw. Technol., vol. 13, pp. 1340-1348, July 1995.

[6] R. Bernini, A. Minardo, and L. Zeni, "Long-range distributed Brillouin fiber sensors by use of an unbalanced double sideband probe," Opt. Express, vol. 19, pp. 23845-23856, November 2011.

[7] A. Zadok, A. Eyal, and M. Tur, "Gigahertz-wide optically reconfigurable filters using stimulated Brillouin scattering” J. Lightw. Technol., vol. 25, pp. 2168-2174, August 2007. 
IEEE copyright 2014 